\title{
Epiglottic Collapse in Obstructive Sleep Apnea
}

\author{
Mi Rye Bae, MD'1, Yoo-Sam Chung, MD, PhD² \\ 'Department of Otolaryngology-Head and Neck Surgery, Bundang Jesaeng General Hospital, Daejin Medical Center, Seongnam, Korea \\ ${ }^{2}$ Department of Otolaryngology-Head and Neck Surgery, Asan Medical Center, University of Ulsan College of Medicine, Seoul, Korea
}

Received: May 7, 2021

Accepted: May 24, 2021

Correspondence

Yoo-Sam Chung, MD, PhD

Department of Otolaryngology-Head

and Neck Surgery,

Asan Medical Center,

University of Ulsan College of Medicine,

88 Olympic-ro 43-gil, Songpa-gu,

Seoul 05505, Korea

Tel +82-2-3010-3710

Fax +82-2-489-2773

E-mail yschung@amc.seoul.kr

ORCID

Mi Rye Bae

https://orcid.org/0000-0002-5683-2178

Yoo-Sam Chung

https://orcid.org/0000-0002-8866-5415

(c) This is an Open Access article distributed under the terms of the Creative Commons Attribution Non-Commercial License (https://creativecommons.org/licenses/by-nc/4.0) which permits unrestricted non-commercial use, distribution, and reproduction in any medium, provided the original work is properly cited.
Along with the development of diagnostic techniques, many studies have been conducted to find the anatomical causes of obstructive sleep apnea (OSA). The velum, oropharynx, tongue base, and epiglottis have been widely considered to be the common obstruction sites. However, the role of the epiglottis in sleep apnea is poorly understood compared to the other anatomical sites. The epiglottis causes OSA either alone or simultaneously with other obstruction sites. We have here reviewed the epidemiology, pathophysiology, diagnosis, and treatment of epiglottic collapse in patients with OSA based on the literature published to date.

Sleep Med Res 2021;12(1):15-19

Key Words Obstructive sleep apnea, Epiglottis, Sleep apnea syndromes.

\section{INTRODUCTION}

Obstructive sleep apnea (OSA) is the periodic decrease or cessation of airflow during sleep due to an upper respiratory tract obstruction. OSA is a relatively common disorder with a prevalence of $1-2 \%$ of the total population, but with a higher incidence in middle age of $4 \%$ in men and $2 \%$ in women [1]. Untreated OSA is associated with an increased risk of cardiovascular disease, cognitive impairment, metabolic disease, and mortality [2].

First line treatments include continuous positive airway pressure (CPAP) but this approach has a poor long-term compliance of $46-85 \%$ and patients often refuse to use it initially [3]. Secondary OSA treatments can include surgery and oral devices. Surgery has proven to be an effective option for reducing cardiovascular morbidity and mortality [4]. Obstruction may occur in all sites of the upper respiratory tract in OSA cases, usually in the soft palate, tongue base and epiglottis. Identifying the obstruction sites and determining the appropriate surgical method increase the success rate of treatments [5].

Drug induced sleep endoscopy (DISE) is also now being used as a new diagnostic tool for selecting therapeutic strategies for OSA [6]. DISE has improved our understanding of the many factors involved in this disorder, one of which is epiglottic collapse. The role of the epiglottis in OSA has been underestimated over the past few decades, but its importance in OSA has emerged and the interests in treatments that target this region have grown in recent years. In our present article, we review the current literature on the epidemiology, pathophysiology, diagnosis, and treatment of epiglottic collapse in OSA patients.

\section{EPIDEMIOLOGY}

Depending on its direction and extent, epiglottic collapse is divided into a partial or complete anteroposterior (A-P) or lateral collapse. Most instances of epiglottic collapse in OSA are a complete A-P collapse, with a rate ranging from $16.8-65.4 \%$. This is followed by a partial A-P col- 
lapse, with a frequency ranging from $3.8-26.1 \%$. The earliest studies of epiglottic collapse involved an analysis of the hypopharynx and larynx using a flexible endoscope when the patient was awake. According to a study by Catalfumo et al. [7] involving an awake endoscopy on 104 persistent OSA patients who received uvulopalatopharyngoplasty (UPPP), $11.5 \%$ of the cases had an abnormally positioned epiglottis.

As DISE became popular, many studies were conducted to identify the frequent areas of upper respiratory tract obstruction using this method. The reported prevalence of epiglottic obstruction in OSA patients has varied from study to study, ranging from $12.5-73.5 \%$. In a 2015 report by Lan et al. [8], the authors noted $12.5 \%$ and $26.6 \%$ of their series of 64 OSA patients had a partial and complete A-P collapse, respectively. These authors found no cases of partial lateral epiglottic collapse in their cohort, but 2 patients (3.1\%) showed a complete lateral collapse. Koutsourelakis et al. [9] reported that among 49 OSA patients assessed using DISE prior to upper respiratory surgery, 36 cases $(73.5 \%)$ experienced some degree of epiglottis collapse. In the responder group in that report, i.e., cases with a post-operative apnoea-hypopnoea index (AHI) of less than 10 that was at least a $50 \%$ decrease from the baseline, $43.4 \%$ of the patients had a complete A-P collapse, $26.1 \%$ had a partial A-P collapse and $4.3 \%$ had a lateral epiglottic collapse. In the non-responder group, $65.4 \%$ of the patients experienced a complete A-P collapse, $3.8 \%$ had a partial A-P collapse, and 3.7\% had a lateral collapse. A recent study using DISE of 324 patients with both nonapneic snoring and OSA reported a floppy epiglottis (FE) in 60 of the subject patients (18.5\%) [10]. In the position dependent group in that report, $19.4 \%$ of the patients had a complete A-P collapse and in the non-position dependent group, $16.8 \%$ of patients had a complete A-P collapse [10]. Notably, however, no significant correlation was found by the authors between the presence of epiglottic collapse and the presence of position dependence.

Several studies have also been conducted using DISE to find remaining obstructions in OSA patients who have not responded to CPAP or surgery. In one retrospective study of 30 cases of failed CPAP with an AHI of less than 5, 11 patients (37\%) showed a persistent A-P collapse, including a collapse at the velum, tongue base, or epiglottis level and a multilevel collapse, and eight patients (27\%) in that study had a FE [11]. Kim et al. [12] evaluated their DISE findings in patients with persistent OSA after UPPP surgery. Of the 50 patients included in that study series with persistent OSA, 22 (44\%) cases had partial or complete epiglottic collapse. In summarizing the studies mentioned above, it appears that the prevalence of epiglottic collapse in OSA patients who fail airway surgery ranges from $44-72.9 \%$.

Isolated epiglottic collapse refers to an A-P collapse other than a lateral or secondary epiglottic collapse of a multilevel area. Few studies to date, however, have investigated this type of collapse. Golz et al. [13] found that 48 out of the 187 OSA patients they examined using nasopharyngoscopy during sleep had retrodis- placement of the epiglottis. Of these cases, 27 (14.4\%) had an isolated epiglottis collapse, whereas 21 had an airway collapse at other sites. Woodson [14] conducted DISE in 117 OSA patients and reported a $16 \%$ rate of isolated obstruction from a ptotic epiglottis. Twelve out of 334 OSA cases (3.5\%) were identified using DISE as an isolated epiglottic collapse group in a prior retrospective analysis of 334 consecutive patients with socially disturbing snoring or symptoms of OSA [15]. In summary, therefore, 3.5\% to $14.4 \%$ of OSA patients show an isolated epiglottic collapse.

Attempts have been made on other studies to identify the obstruction sites in OSA using methods other than DISE or an awake endoscope. In one such report, 48 OSA patients were examined using videofluoroscopy, and epiglottic collapse was observed in 14 cases (29.2\%) [16].

\section{PATHOPHYSIOLOGY}

The underlying pathophysiology of epiglottic collapse is still not well understood. In the current literature, several mechanisms have been proposed to explain this phenomenon including 1) secondary to anterior-posterior collapse of the tongue base that pushes the epiglottis backwards; 2) a complete isolated anterior-posterior epiglottic collapse, also known as the trapdoor phenomenon; and 3) lateral collapse of the epiglottis due to underdevelopment of the epiglottis itself [10]. These are further discussed below.

\section{Secondary to an Anterior-Posterior Collapse of the Tongue Base}

When a bulky tongue base pushes it backwards, the epiglottis blocks the upper airway. Macroglossia, hypotonia, retrognathia, or lingual tonsil hypertrophy can cause the collapse of the tongue base and epiglottis.

\section{Trapdoor Phenomenon}

The trapdoor phenomenon, or FE, refers to a collapsible epiglottis that blocks the airway [17]. The reason for the airway obstruction in this instance is a prolapse of the epiglottis into the posterior pharyngeal wall during strong inspiration. The cause of this phenomenon is unknown, but several possible hypotheses can be considered. First, involuntary swallowing during sleep can cause an epiglottic prolapse in some people. Alternatively, some patients with a weakened glossoepiglottic ligament are more prone to an epiglottis collapse than the general population. An unanswered question in this regard is whether an epiglottis of a particular shape is more prone to collapse. The relationship between the shape of the epiglottis and its potential collapse is still controversial. In a prior case-control study involving 11 isolated epiglottic collapse cases and 44 controls, there were no differences found in the epiglottic shape or curvature between the two groups [15]. In another study, however, in which an esophagogastrodu- 
odenoscopy was performed in more than 50 patients, the authors identified a positive association between a concave posterior surface of the epiglottis and the degree of airway collapse, and consequently the severity of OSA [17].

\section{Underdevelopment of the Epiglottis}

Laryngomalacia is the most common cause of stridor in infants. In these patients, the epiglottis can easily block the upper airway and cause apnea. Verkest et al. [18] reported a 77\% prevalence of OSA in children with laryngomalacia. The proposed mechanisms to date include 1) a delayed maturation or "hypotonia" of the supporting cartilaginous structures of the larynx; 2) redundant soft tissue in the supraglottis; and 3) a foreshortened or aryepiglottic fold [19-21]. Although laryngomalacia resolves with the growth and development of a child, acquired laryngomalacia in adults has also been reported. Adult laryngomalacia is broadly classified into idiopathic $[13,17]$ or postoperative and post-traumatic secondary cases [19]. The mechanism of idiopathic adult laryngomalacia has not been fully elucidated.

\section{DIAGNOSIS}

Friedman staging, cephalometry, CT/MR, Müller's maneuver using a flexible endoscope, and intrapharyngeal pressure manometry are the most widely used diagnostic tools to identify obstruction sites in patients with OSA. The Müller's maneuver using a flexible endoscope has the advantage of being simple, economical, minimally invasive, and capable of checking the dynamic upper respiratory tract. It is, thus, a commonly used approach in OSA patients to identify the obstruction sites. The Müller's maneuver method has the disadvantage, however, of low reproducibility and range of interpretations among different examiners. Moreover, all of the tests mentioned above are limited by their inability to accurately represent the obstruction that occurs during sleep because they are conducted while the patient is awake [20,21].

Since Croft and Pringle [22] first described "sleep nasoendoscopy during sedation" in 1991, DISE has been widely used to determine the exact site of the upper airway collapse in patients with OSA. The biggest advantage of DISE is that it allows the direct observation of the obstruction in the upper respiratory tract during sleep $[5,6]$. This technique has, thus, proven to be a useful method of assessing the location, severity, and pattern of airway obstruction. The velum, oropharynx, tongue base, epiglottis (VOTE) classification is a standardized method for evaluating DISE results and shows excellent intra- and inter-rater reliability [6]. DISE also has several drawbacks, however. First, it is conducted under conditions of pharmacologically induced sleep, not natural sleep. Second, DISE mainly reflects stage 2 sleep i.e., not the entire stages of sleep. Third, it is difficult to simultaneously evaluate the oropharynx and tongue base with DISE. How- ever, DISE is currently the gold standard tool for assessing OSA.

Videofluoroscopy is a method in which the patient is placed in a supine position to perform induced sleep, and the lateral side is then photographed via fluoroscopy to identify the obstruction site. This method also has some limitations, however. First, it measures only the lateral side and only anterior or posterior obstructions can be checked. Second, it is performed during induced sleep only, as is the case with DISE. However, it is a valuable noninvasive method of observing the entire upper airway tract [16].

Azarbarzin et al. [23] conducted a trial in 2017 to detect distinct airflow patterns in patients with epiglottic collapse. In that study, epiglottic collapse was characterized by a rapid fall in the inspiratory flow, more variable inspiratory and expiratory flow, and a reduced tidal volume. The usefulness of these flow characteristics has not yet been verified, but this test can be performed concurrently with polysomnography during natural sleep, and may thus become widely used in the future.

\section{TREATMENT}

\section{CPAP}

Interest in epiglottic collapse in OSA has been increasing in recent years, and a variety of therapeutic options have been attempted to resolve this condition. CPAP, the standard treatment method for OSA, has also been attempted, but has shown limited efficacy [15]. The role of CPAP is to stent the upper airway through the application of continuous positive pressure. However, in the case of primary epiglottis collapse, CPAP can exacerbate the airway obstruction by pushing the epiglottis down into the laryngeal inlet $[24,25]$. There are also some case reports that CPAP failure is related to epiglottic collapse [26].

In a study published by Shimohata et al. [27], the authors reported their results for 17 patients displaying multiple system atrophy during DISE, 12 of whom showed a FE. None of the patients with severe FE $(n=3)$ improved with CPAP treatment. In patients with mild FE $(n=9)$, CPAP caused more severe FE during inspiration, reducing the oxygen saturation levels in 2 cases and improved airway obstruction in the remaining 7 patients [25,27]. There have been some case reports of infants with laryngomalacia treated with CPAP [28]. Except for a few instances, however, CPAP generally aggravates the epiglottic collapse, making it problematic to use as a therapeutic method in OSA.

\section{Positional Therapy and Oral Devices}

There are several reports on the effects of positional therapy and the use of oral devices to treat an epiglottic collapse. A previous case-control study of 224 patients by Kim et al. [29] compared the clinical characteristics of cases with and without an epiglottic collapse (epi group vs. non-epi group). The epi group cases in that report showed a significantly lower body mass index and OSA severity. In addition, the epi cases were expected 
to respond well to the effects of oral devices or positional therapy. Kent et al. [30] reported on the outcomes in 11 out of 35 patients with epiglottic obstruction after oral appliance therapy. Seven of these patients showed persistent epiglottic obstruction, whereas the symptoms improved in the remaining four cases. According to findings of Sung et al. [15], of 11 patients with isolated epiglottic collapse in their study series, 9 cases (81.8\%) could be resolved with a mouth open and jaw thrust maneuver, which simulated the use of an oral appliance. Six cases in that study (54.4\%) were resolved by turning their heads to mimic the lateral sleeping posture.

Vonk et al. [10] reported from their study that only 4 out of 60 patients with a FE had a persistence of this condition when performing a lateral head rotation. A FE was found in only one subject when the patient was tilted to the side of the head and torso. The FE still existed in 10 of these patients after applying the jaw thrust maneuver. As such, positional therapy and oral devices have been reported to have some effect in alleviating epiglottic collapse, but the research is still limited in this regard and further validation is needed.

\section{Epiglottis Surgery}

\section{Partial epiglottectomy}

A partial epiglottectomy is a useful treatment for a trapdoor epiglottis and laryngomalacia. The procedure can be performed in various ways, and the epiglottis is excised using a laser or monopolar coagulator. Catalfumo et al. [7] described their use of an endoscopic $\mathrm{CO}_{2}$ laser partial epiglottectomy. The results of that study indicated that a partial epiglottectomy can increase the cure rate of patients with OSA by 10-15\%. In 2001, a partial epiglottectomy using monopolar diathermy was described by Oluwasanmi and Mal [31] who reported four cases treated in this way because of snoring and/or OSA due partly or wholly to a FE.

Golz et al. [13] published the results of a laser partial epiglottectomy in 2000 as a treatment for OSA and laryngomalacia in which a U-shaped wedge is excised in the suprahyoid portion of the epiglottis. Polysomnography performed postoperatively in adult OSA patients showed statistically significant improvement in $85 \%$ of cases with this therapy. In all of the cases of laryngomalacia treated with this method, the stridor was permanently stopped after surgery with complete cessation of the apneic episodes.

Excessive resection of the epiglottis can lead to aspiration, and insufficient resection runs the risk of being ineffective against OSA. It is difficult to properly determine the volume of epiglottis to cut without causing postoperative sequelae. Bartolomeo et al. [32] described their experiences with a 25-year-old man with laryngomalacia managed using $\mathrm{CO}_{2}$ laser $\mathrm{V}$-shape partial epiglottectomy. The authors stated that this treatment minimizes the risk of false passage, while ensuring permanent respiratory airflow through the epiglottic $\mathrm{V}$ during epiglottic movement.

\section{Epiglottis stiffening operation}

The epiglottis stiffening operation was first described in 2019 by Salamanca et al. [24]. In this procedure, the lower half of the lingual side of the epiglottis is cauterized in the area between the lateral glosso-epiglottic folds using suction cautery. This induces stiffening and scar retraction of tissues as a result of secondary healing. There were no patients with complications such as dysphagia or aspiration dysphonia during a follow-up period of between 3 and 15 months.

\section{Tongue Base Advancement}

\section{Hyoid suspension (hyothyroidopexy)}

Hyoid suspension represents another surgical option for the treatment of epiglottic collapse. Kezirian [33] reported that none of their patients with a suspended hyoid developed epiglottic collapse. According to a prior meta-analysis by Song et al. [34], isolated hyoid surgery reduces the severity of OSA and improves sleepiness in adults. In that review, hyothyroidopexy was reported to reduce AHI by the greatest amount (50.7\%), followed by hyoid myotomy with suspension (a 38.3\% reduction in AHI) and hyoid expansion (a 7.1\% reduction in AHI). Askar et al. [35] performed a voice and swallowing assessment in 17 patients who had undergone hyothyroidpexy. Their comparative analysis of the pre- and post-operative results of voice and swallowing measures revealed a non-significant difference. Hyoid suspension is, thus, a theoretically possible surgical option in OSA, but there are no studies that have proven its direct effect on epiglottis collapse.

\section{Genioglossus advancement}

The genioglossus muscle is the most important muscle for maintaining airway patency during sleep. Genioglossus advancement, first introduced in 1984 by Riley et al. [36], increases the muscle tension of the tongue and limits posterior collapse during sleep. Several studies have demonstrated that genioglossus advancement combined with an UPPP achieves an improvement in OSA patients. Complications from this surgery may include hematoma, injury to the genioglossus muscle, and abnormal sensation of the mandible. There is a possibility also that an epiglottic collapse will improve as the tongue base advances, but there is no current research on this.

\section{CONCLUSION}

Traditionally, the velum and oropharynx have been considered the main sites of obstruction in OSA patients. As diagnostic tools such as DISE have become popular for upper airway tract examinations during sleep, the critical role of the epiglottis in OSA has been revealed. The prevalence of epiglottic collapse in patients with OSA is likely to be higher than previously described. If there is residual obstruction even after CPAP or 
UPPP surgery, an upper respiratory reassessment should be performed to determine the presence and type of epiglottic collapse. Personalized treatments for OSA are, thus, warranted, based on the type of collapse.

\section{Acknowledgments}

None.

\section{Conflicts of Interest}

The authors have no financial conflicts of interest.

\section{Authors' Contribution}

Conceptualization: Chung YS. Data curation: all authors. Supervision: Chung YS. Writing—original draft: Bae MR. Writing—review \& editing: Chung YS.

\section{REFERENCES}

1. Young T, Palta M, Dempsey J, Skatrud J, Weber S, Badr S. The occurrence of sleep-disordered breathing among middle-aged adults. $N$ Engl J Med 1993;328:1230-5.

2. Shahar E, Whitney CW, Redline S, Lee ET, Newman AB, Nieto FJ, et al. Sleep-disordered breathing and cardiovascular disease: cross-sectional results of the Sleep Heart Health Study. Am J Respir Crit Care Med 2001;163:19-25.

3. Sin DD, Mayers I, Man GC, Pawluk L. Long-term compliance rates to continuous positive airway pressure in obstructive sleep apnea: a population-based study. Chest 2002;121:430-5.

4. Certal V, Nishino N, Camacho M, Capasso R. Reviewing the systematic reviews in OSA surgery. Otolaryngol Head Neck Surg 2013;149: 817-29.

5. Stuck BA, Maurer JT. Airway evaluation in obstructive sleep apnea. Sleep Med Rev 2008;12:411-36.

6. Kezirian EJ, Hohenhorst W, de Vries N. Drug-induced sleep endoscopy: the VOTE classification. Eur Arch Otorhinolaryngol 2011;268:1233-6.

7. Catalfumo FJ, Golz A, Westerman ST, Gilbert LM, Joachims HZ, Goldenberg D. The epiglottis and obstructive sleep apnoea syndrome. $J$ Laryngol Otol 1998;112:940-3.

8. Lan MC, Liu SY, Lan MY, Modi R, Capasso R. Lateral pharyngeal wall collapse associated with hypoxemia in obstructive sleep apnea. Laryngoscope 2015;125:2408-12.

9. Koutsourelakis I, Safiruddin F, Ravesloot M, Zakynthinos S, de Vries N. Surgery for obstructive sleep apnea: sleep endoscopy determinants of outcome. Laryngoscope 2012;122:2587-91.

10. Vonk PE, Ravesloot MJL, Kasius KM, van Maanen JP, de Vries N. Floppy epiglottis during drug-induced sleep endoscopy: an almost complete resolution by adopting the lateral posture. Sleep Breath 2020;24: 103-9.

11. Dieleman E, Veugen CCAFM, Hardeman JA, Copper MP. Drug-induced sleep endoscopy while administering CPAP therapy in patients with CPAP failure. Sleep Breath 2021;25:391-8.

12. Kim DK, Lee JW, Lee JH, Lee JS, Na YS, Kim MJ, et al. Drug induced sleep endoscopy for poor-responder to uvulopalatopharyngoplasty in patient with obstructive sleep apnea patients. Korean J Otorhinolaryngol-Head Neck Surg 2014;57:96-102.

13. Golz A, Goldenberg D, Westerman ST, Catalfumo FJ, Netzer A, Westerman LM, et al. Laser partial epiglottidectomy as a treatment for obstructive sleep apnea and laryngomalacia. Ann Otol Rhinol Laryngol 2000;109:1140-5.

14. Woodson BT. A method to describe the pharyngeal airway. Laryngoscope 2015;125:1233-8.

15. Sung CM, Kim HC, Yang HC. The clinical characteristics of patients with an isolate epiglottic collapse. Auris Nasus Larynx 2020;47:450-7.

16. Choi HR, Han K, Lee J, Hong SC, Kim JK, Cho JH. Analysis of ob- struction site in obstructive sleep apnea patients based on videofluoroscopy. J Rhinol 2019;26:21-5.

17. Kanemaru S, Kojima H, Fukushima H, Tamaki H, Tamura Y, Yamashita $\mathrm{M}$, et al. A case of floppy epiglottis in adult: a simple surgical remedy. Auris Nasus Larynx 2007;34:409-11.

18. Verkest V, Verhulst S, Van Hoorenbeeck K, Vanderveken O, Saldien V, Boudewyns A. Prevalence of obstructive sleep apnea in children with laryngomalacia and value of polysomnography in treatment decisions. Int J Pediatr Otorhinolaryngol 2020;137:110255.

19. Woo P. Acquired laryngomalacia: epiglottis prolapse as a cause of airway obstruction. Ann Otol Rhinol Laryngol 1992;101:314-20.

20. Li W, Ni D, Jiang H, Zhang L. [Predictive value of sleep nasendoscopy and the Müller maneuver in uvulopalatopharyngoplasty for the obstructive sleep apnea syndrome]. Lin Chuang Er Bi Yan Hou Ke Za Zhi 2003;17:145-6.

21. Hori Y, Shizuku H, Kondo A, Nakagawa H, Kalubi B, Takeda N. Endoscopic evaluation of dynamic narrowing of the pharynx by the Bernouilli effect producing maneuver in patients with obstructive sleep apnea syndrome. Auris Nasus Larynx 2006;33:429-32.

22. Croft CB, Pringle M. Sleep nasendoscopy: a technique of assessment in snoring and obstructive sleep apnoea. Clin Otolaryngol Allied Sci 1991;16:504-9.

23. Azarbarzin A, Marques M, Sands SA, Op de Beeck S, Genta PR, Taranto-Montemurro L, et al. Predicting epiglottic collapse in patients with obstructive sleep apnoea. Eur Respir J 2017;50:1700345.

24. Salamanca F, Leone F, Bianchi A, Bellotto RGS, Costantini F, Salvatori P. Surgical treatment of epiglottis collapse in obstructive sleep apnoea syndrome: epiglottis stiffening operation. Acta Otorhinolaryngol Ital 2019;39:404-8.

25. Torre C, Camacho M, Liu SY, Huon LK, Capasso R. Epiglottis collapse in adult obstructive sleep apnea: a systematic review. Laryngoscope 2016; 126:515-23.

26. Sanches I, Martins V, Santos JMD. Obstructive sleep apnea refractory to treatment due to floppy epiglottis. Arch Bronconeumol 2015;51:94.

27. Shimohata T, Shinoda H, Nakayama H, Ozawa T, Terajima K, Yoshizawa $\mathrm{H}$, et al. Daytime hypoxemia, sleep-disordered breathing, and laryngopharyngeal findings in multiple system atrophy. Arch Neurol 2007;64:856-61.

28. Zwacka G, Scholle S, Kemper G, Rieger B. Nasal CPAP therapy for infants with congenital stridor. Sleep Breath 1997;2:85-97.

29. Kim HY, Sung CM, Jang HB, Kim HC, Lim SC, Yang HC. Patients with epiglottic collapse showed less severe obstructive sleep apnea and good response to treatment other than continuous positive airway pressure: a case-control study of 224 patients. J Clin Sleep Med 2021;17:413-9.

30. Kent DT, Rogers R, Soose RJ. Drug-induced sedation endoscopy in the evaluation of OSA patients with incomplete oral appliance therapy response. Otolaryngol Head Neck Surg 2015;153:302-7.

31. Oluwasanmi AF, Mal RK. Diathermy epiglottectomy: endoscopic technique. J Laryngol Otol 2001;115:289-92.

32. Bartolomeo M, Bigi A, Pelliccia P, Makeieff M. Surgical treatment of a case of adult epiglottic laryngomalacia. Eur Ann Otorhinolaryngol Head Neck Dis 2015;132:45-7.

33. Kezirian EJ. Nonresponders to pharyngeal surgery for obstructive sleep apnea: insights from drug-induced sleep endoscopy. Laryngoscope 2011;121:1320-6.

34. Song SA, Wei JM, Buttram J, Tolisano AM, Chang ET, Liu SY, et al. Hyoid surgery alone for obstructive sleep apnea: a systematic review and meta-analysis. Laryngoscope 2016;126:1702-8.

35. Askar SM, Quriba AS, Hassan EM, Awad AM, Bessar AA. Voice and swallowing outcomes after hyoid suspension surgery in patients with obstructive sleep apnea. Folia Phoniatr Logop 2017;69:271-7.

36. Riley R, Guilleminault C, Powell N, Derman S. Mandibular osteotomy and hyoid bone advancement for obstructive sleep apnea: a case report. Sleep 1984;7:79-82. 